\title{
A simple double-focusing electrostatic ion beam deflector
}

\author{
H. Kreckel, ${ }^{1, a)}$ H. Bruhns, ${ }^{1, b)}$ K. A. Miller, ${ }^{1}$ E. Wåhlin, ${ }^{2}$ A. Davis, ${ }^{1, c)}$ S. Höckh, ${ }^{1, d)}$ \\ and D. W. Savin ${ }^{1}$ \\ ${ }^{1}$ Columbia University, 550 West 120th Street, New York, New York 10027, USA \\ ${ }^{2}$ Beam Imaging Solutions, 1610 Pace Street, Unit 900 \#303, Longmont, Colorado 80504, USA
}

(Received 22 February 2010; accepted 1 May 2010; published online 28 June 2010)

\begin{abstract}
We have developed an electrostatic, double-focusing $90^{\circ}$ deflector for fast ion beams consisting of concentric cylindrical plates of differing heights. In contrast to standard cylindrical deflectors, our design allows for focusing of an incoming parallel beam not only in the plane of deflection but also in the orthogonal direction. The optical properties of our design resemble those of a spherical capacitor deflector while it is much easier and more cost effective to manufacture. (C) 2010 American Institute of Physics. [doi:10.1063/1.3433485]
\end{abstract}

\section{INTRODUCTION}

Charged particle beams are used in a wide variety of environments, ranging from fundamental research to industrial applications and cancer treatment. Most of the early developments in ion optics were motivated by electron beam studies, ${ }^{1}$ and since the advent of ion sources, an array of electron and ion beam deflectors has been developed. ${ }^{2,3}$ While magnetic deflectors and lenses are commonly used at high energies $(>50 \mathrm{keV})$, electrostatic elements have advantages in the low energy to mid energy range $(1-50 \mathrm{keV})$ due to their lower cost, ease of construction, and the absence of hysteresis. Five different types of electrostatic ion beam deflectors are used routinely in laboratories: the parallel plate mirror analyzer, ${ }^{4}$ the cylindrical mirror analyzer, ${ }^{5}$ the radial cylindrical analyzer, ${ }^{6}$ the spherical capacitor, ${ }^{7}$ and the quadrupole deflector. ${ }^{8}$

In many experiments, beam deflectors are used as energy analyzers or monochromators and precise construction and detailed understanding of the optical properties are required. ${ }^{9}$ On the other hand, there are also applications where beam deflectors are used to simply bend an ion beam by $90^{\circ}$ in order to facilitate differential pumping or background suppression or to direct ions toward a detector. In this publication, we describe a variation in the radial cylindrical analyzer that we use to deflect $\mathrm{keV}$ energy ion beams by right angles. Compared to a standard cylindrical analyzer, our design has the advantage that it focuses an incoming parallel beam not only in the dispersion plane but also in the orthogonal direction. This is achieved by using cylindrical sector plates which differ in height. ${ }^{10}$ When properly implemented, our

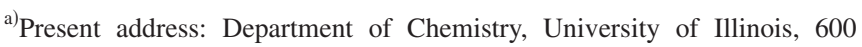
South Mathews Avenue, Urbana, IL 61801, USA. Electronic mail: hkreckel@illinois.edu.

${ }^{b)}$ Present address: Inficon GmbH, D-50968 Cologne, Germany.

${ }^{c}$ Present address: Curtis High School, 105 Hamilton Avenue, Staten Island, NY 10301, USA.

${ }^{d)}$ Present address: Eberhard-Karls-Universität Tübingen, GeschwisterScholl-Platz, 72074 Tübingen, Germany.
}

deflector has ion-optical properties similar to those of a spherical capacitor, while it is much easier to assemble and less costly.

In many experimental setups, electrostatic quadrupole deflectors are used to deflect ion beams by right angles. ${ }^{11}$ However, in order to avoid asymmetric beam profiles, often elaborately placed shim electrodes have to be introduced. ${ }^{8}$ Furthermore, quadrupoles need to be supplied with voltages almost as high as the ion beam energy (in units of eV), requiring expensive high voltage feedthroughs and power supplies for beam energies $>5 \mathrm{keV}$. Our deflector design can be used with voltages corresponding to $\sim 1 / 4$ of the ion beam energy, making it much easier to provide the required potentials.

\section{PRINCIPLE OF OPERATION}

Using the SIMION 3D program suite, ${ }^{12,13}$ we have simulated the $90^{\circ}$ deflection of a collimated $10 \mathrm{keV}$ ion beam of singly negatively charged particles by a radial cylindrical deflector. The results are shown in Fig. 1. The deflector consists of two concentric cylindrical electrode sectors of the same height (with radii of $R_{1}=140 \mathrm{~mm}$ and $R_{2}=178 \mathrm{~mm}$, respectively ${ }^{14}$ ) and two aperture plates, defining the entrance and exit of the deflector and reducing fringe field effects. A circular ion beam of $6 \mathrm{~mm}$ diameter enters the structure midway between the electrodes through the $12 \mathrm{~mm}$ wide aperture.

The potential difference that is necessary to guide an ion with energy $U_{\text {beam }}$ on the designated center radius $R_{0}$ between the cylindrical plates can be calculated by equating the electrostatic force and the centrifugal force,

$$
q E=\frac{m v^{2}}{R_{0}}
$$

with $E$ being the electric field strength, $v$ being the velocity of the particle, $m$ the mass, and $q$ the charge. Substituting the kinetic energy $U_{\text {beam }}=(1 / 2) m v^{2}$ on the right-hand side and expressing terms in units of $\mathrm{eV}$ yield the relation 


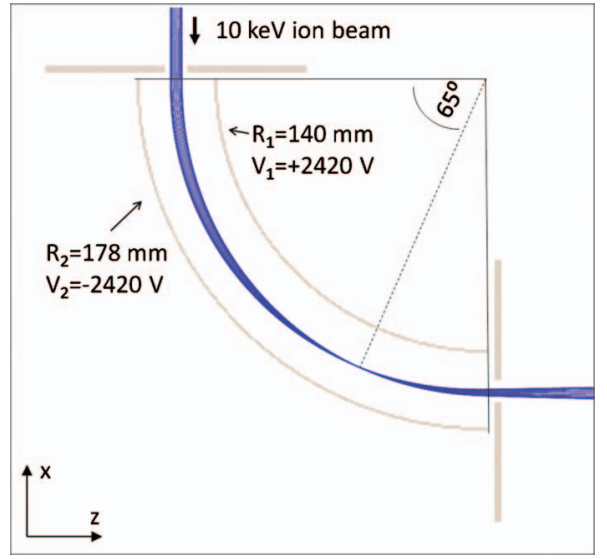

FIG. 1. (Color) SIMION 3D simulation of the deflection of a singly negatively charged ion beam of $10 \mathrm{keV}$ energy in a radial cylindrical $90^{\circ}$ deflector. The dashed line marks the position of narrowest beam waist.

$$
E[\mathrm{~V} / \mathrm{m}]=\frac{2 U_{\text {beam }}[\mathrm{eV}]}{R_{0}[\mathrm{~m}]} .
$$

For the simulated negative ion beam of $10 \mathrm{keV}$ energy and $R_{0}=159 \mathrm{~mm}$, the required field strength is $125.8 \mathrm{kV} / \mathrm{m}$, resulting in a potential difference of $4780 \mathrm{~V}$ for the two cylindrical plates which are $38 \mathrm{~mm}$ apart. In reality, the incoming particles undergo a smooth transition, from an infinite radius to $R_{0}$ and back, upon entrance and exit of the deflector, and depending on the aperture design, the potential values can vary slightly. In the simulation shown here, potentials of $\pm 2420 \mathrm{~V}$ were applied to the inner/outer plate.

In the $x z$-dispersion plane, the ion beam experiences a focusing force that results in a narrow beam waist after a deflection angle of $\sim 65^{\circ}$. For an ideal cylindrical analyzer, one would expect the focus to occur after $\sim 63.5^{\circ}$ since refocusing of a spot-sized incoming beam occurs at a deflection angle of $\pi / \sqrt{2}=127^{\circ}{ }^{6}$

In conventional cylindrical deflectors, no electric field acts upon the ions perpendicular to the deflection plane; hence a collimated ion beam will retain its parallelism and beam size in the $y$ direction. In the dispersion plane, the emerging beam is divergent and in order to collimate it again an asymmetric focusing element would be required. For a spherical capacitor deflector, on the other hand-consisting of a pair of concentric spheres-refocusing occurs in the deflection plane as well as in the $y$ direction after $180^{\circ}$. Consequently, a collimated beam entering a $90^{\circ}$ spherical capacitor is focused into a spot at the exit aperture and can be collimated again by an Einzel lens.

Ignoring the beam deflection, the optical analog to the cylindrical deflector is a cylindrical lens, while the spherical deflector acts as a spherical lens. Figure 2(a) shows the cross section of a spherical capacitor with the same plate radii (140 and $178 \mathrm{~mm})$ and voltages $( \pm 2420 \mathrm{~V})$ used for the cylindrical deflector simulation shown in Fig. 1. In the central part that is of interest for ion transport, the equipotential lines follow the curvature of the plates closely. The focusing in the $y$ direction is caused by this curvature of the field lines between the deflection plates. Figure 2(b) shows the cross section of cylindrical plates with differing plate heights of 45
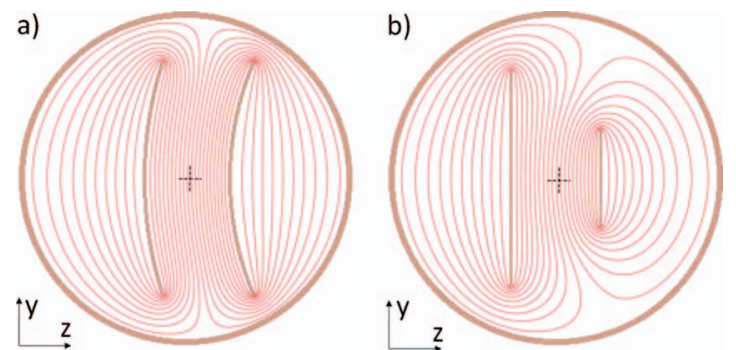

FIG. 2. (Color) Comparison of the equipotential lines in the cross section of (a) a spherical capacitor and (b) a cylindrical capacitor with differing electrode heights of 101 and $45 \mathrm{~mm}$. For the spherical capacitor, the inner (outer) plate has a radius of $140 \mathrm{~mm}(178 \mathrm{~mm})$. The potentials applied to the electrodes are the same for both examples, with $2420 \mathrm{~V}$ on the inner electrode and $-2420 \mathrm{~V}$ on the outer electrode. To define the boundary conditions, the electrodes are surrounded by grounded beam pipes $(152 \mathrm{~mm}$ inner diameter). The black cross hairs mark the central region that is of interest for the transport of ions.

and $101 \mathrm{~mm}$ for the inner and outer plates, respectively. With the same voltages applied, the fields in the central part between the electrodes (marked by a cross hair) exhibit a slight curvature similar to those seen in the spherical capacitor. It is this resemblance in the field geometry that we exploit to achieve focusing in the $x z$ plane as well as in the $y$ direction, utilizing cylindrical deflector plates with differing heights.

Figure 3 shows the three-dimensional SIMION simulation of a cylindrical deflector with differing plate heights $(45 \mathrm{~mm}$ for the inner electrode and $101 \mathrm{~mm}$ for the outer electrode). The deflector was designed to fit into a standard DN160 CF
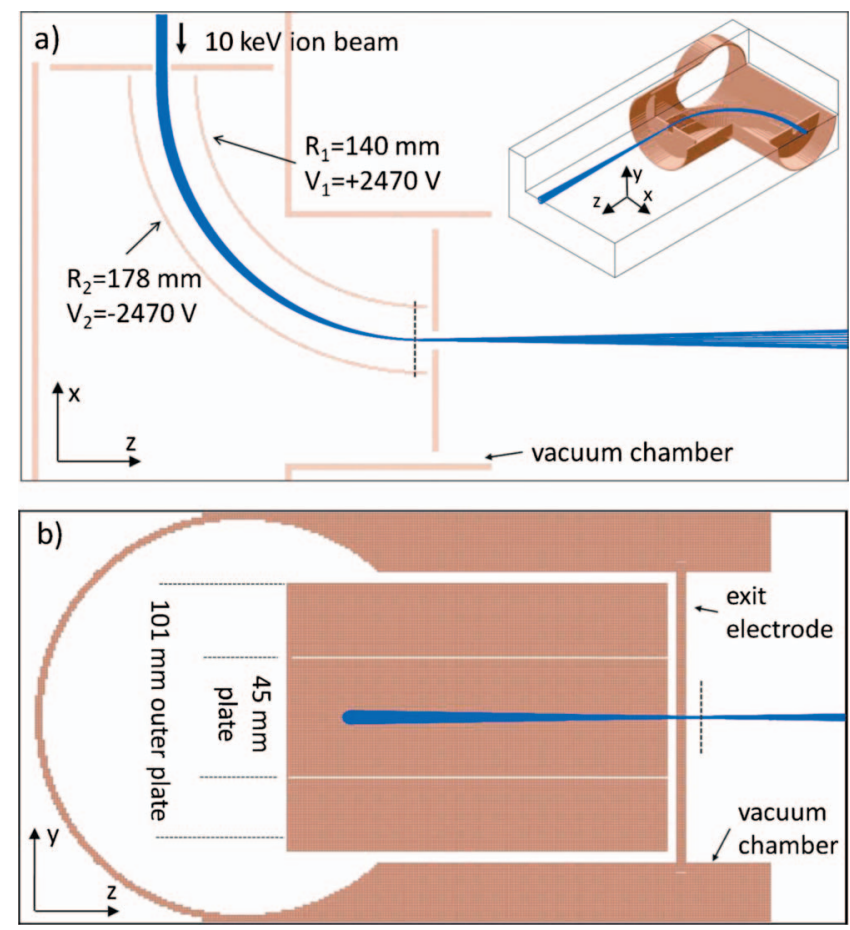

FIG. 3. (Color) SIMION 3D simulation of the deflection of a $10 \mathrm{keV}$ ion beam in a cylindrical $90^{\circ}$ deflector with differing plate heights. The entrance and exit apertures are grounded. In (a) we show the beam trajectories in the dispersion plane; the inset depicts an isometric view of the deflector inside the vacuum chamber which is modeled to have inner dimensions corresponding to a standard DN160 CF-Tee. In (b) the focusing in the $y$ direction can be seen. The positions of narrowest beam waist in each plane are marked by dashed lines. 

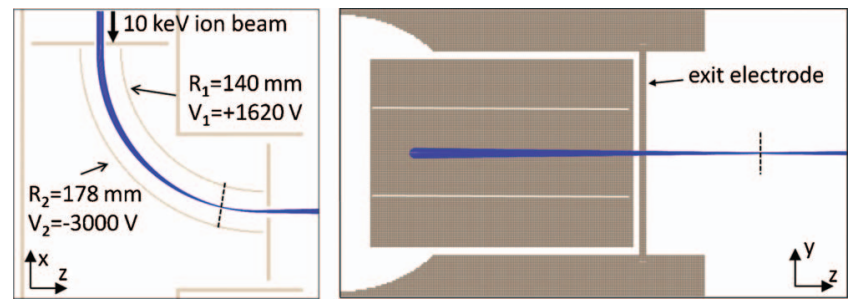

FIG. 4. (Color) SIMION 3D simulation of the deflection of a $10 \mathrm{keV}$ ion beam in a cylindrical $90^{\circ}$ deflector with differing plate heights and asymmetric voltages. The positions of narrowest beam waist are marked by dashed lines.

vacuum chamber. The same electrode radii were used as in the conventional cylindrical deflector depicted in Fig. 1. The smaller plate height for the inner electrode has three immediate effects on the ion trajectories: (i) the net field strength between the plates is weakened and hence slightly higher voltages are needed to guide the ion beam $( \pm 2470 \mathrm{~V}$ as compared to $\pm 2420 \mathrm{~V}$ ); (ii) the focus in the dispersion plane occurs later, it is shifted to about $5 \mathrm{~mm}$ before the exit electrode; (iii) in the $y$ direction, the ion beam is no longer unaffected, a narrow beam waist forms about $3 \mathrm{~mm}$ behind the exit aperture. At the position of the exit aperture, the incoming circular beam of $6 \mathrm{~mm}$ diameter is compressed to $\sim 0.6 \mathrm{~mm}$ in $x$ and $\sim 0.4 \mathrm{~mm}$ in $y$.

In principle it is possible to shift the focus in the deflection plane as well as orthogonal to it by changing the electrode plate height ratio and the geometry of the entrance and exit electrodes. However, practical limitations for the size of the deflector, the opening apertures for the ion beam, and the vacuum chamber housing have to be taken into account. Moreover, the optical properties can be adjusted in a much more flexible fashion when asymmetric voltages are used. In Fig. 4 the same deflector is shown with asymmetric voltages of -3000 and $+1620 \mathrm{~V}$. In this example, the focus in the deflection plane is shifted inside the structure, while the focus in the $y$ direction now lies further downstream of the exit electrode. The potential difference between the two plates for $90^{\circ}$ deflection is reduced to $4620 \mathrm{~V}$. This can be explained by the re-referencing of the kinetic energy that the ions experience when they enter the deflector. Since the voltages applied to the plates are no longer centered around zero, the ions will be decelerated while they are passing through the deflector. Tangential to their flight path, the incoming negatively charged ions lose $(1 / 2)(-3000+1620) \mathrm{eV}=-690 \mathrm{eV}$ of kinetic energy after they enter through the grounded entrance aperture. The remaining kinetic energy amounts to $9.31 \mathrm{keV}$ and according to Eq. (2) we expect a voltage difference of $4450 \mathrm{~V}$. However, since the ions are not accelerated and decelerated abruptly upon entering and leaving the deflector, their actual average energy inside the deflector lies between 10 and $9.31 \mathrm{keV}$. Hence the actual potential difference of $4620 \mathrm{~V}$ needed to deflect the beam ranges between the extreme values of $4940 \mathrm{~V}$ (for a $10 \mathrm{keV}$ beam) and $4450 \mathrm{~V}$ (for a $9.31 \mathrm{keV}$ beam).

From our models, we find that for the given geometry and $2250 \mathrm{~V}(-2620 \mathrm{~V})$ on the inner (outer) plates a narrow beam waist forms just inside the exit aperture, both in the dispersion plane as well as in the $y$ direction. Figure 5 shows

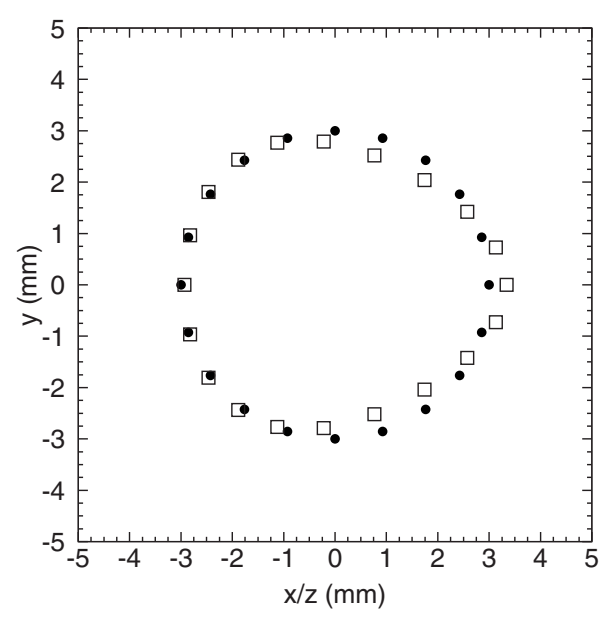

FIG. 5. Simulated beam profiles of an incoming $6 \mathrm{~mm}$ diameter ion beam before the deflector (solid black dots) and $150 \mathrm{~mm}$ behind the exit aperture (open squares). Note that the incoming beam is parallel while the outgoing beam is divergent.

the beam profile of the incoming circular ion beam in comparison to the profile $150 \mathrm{~mm}$ behind the exit aperture. The envelopes are of similar size and aspect ratio; however, the outgoing beam is slightly deformed. For many applications this deformation is not significant, especially since ion sources rarely emit perfectly symmetric ion beams. Note that the incoming beam in Fig. 5 is parallel while the outgoing beam is divergent. For collimated beam transport an appropriately positioned Einzel lens downstream of the deflector is required. It is also possible to send a convergent beam into the deflector in order to achieve a parallel beam at the exit.

\section{PRACTICAL IMPLEMENTATION AND TESTS}

The first version of the asymmetric cylindrical deflector was built with a plate height ratio of $4 \mathrm{inch} / 2$ inch $(101.6 \mathrm{~mm} / 50.8 \mathrm{~mm})$ and radii of 178 and $140 \mathrm{~mm}$, respectively. It was used as an energy filter to separate single 20 $\mathrm{keV} \mathrm{H}_{2}^{+}$ions out of an intense $10 \mathrm{keV}$ proton beam for a merged beams experiment. ${ }^{15}$ For background suppression, two consecutive deflectors were used in order to direct the $\mathrm{H}_{2}^{+}$ions onto a channeltron detector. Tests with a Faraday cup revealed a transmission through both deflectors compatible with unity $(99 \pm 1 \%){ }^{15}$

In order to verify the beam shaping potential of asymmetric voltages, beam profile measurements were performed with a rudimentary test version of the deflector with inner and outer plate radii of 145 and $185 \mathrm{~mm}$, respectively. We used a collimated $\mathrm{H}^{-}$beam at $10 \mathrm{keV}$, extracted from a duoplasmatron ion source and shaped by two $5 \mathrm{~mm}$ circular apertures, approximately $5 \mathrm{~m}$ apart. The result is a uniformly filled circular ion beam of $5 \mathrm{~mm}$ diameter with negligible divergence ( $\leq 1 \mathrm{mrad}$ ). We recorded the beam profiles with a Beam Imaging Solutions Model BOS-18 Beam Observation System based on a microchannel plate (MCP) and a phosphor screen. Figure 6 shows the $\mathrm{H}^{-}$beam profile after deflection at a position $\sim 76 \mathrm{~mm}$ behind the exit aperture. The voltages of the outer/inner plates are given in the insets. The effect that asymmetric voltages have on the position of the foci is clearly visible as the beam shape measured in the 
before deflection
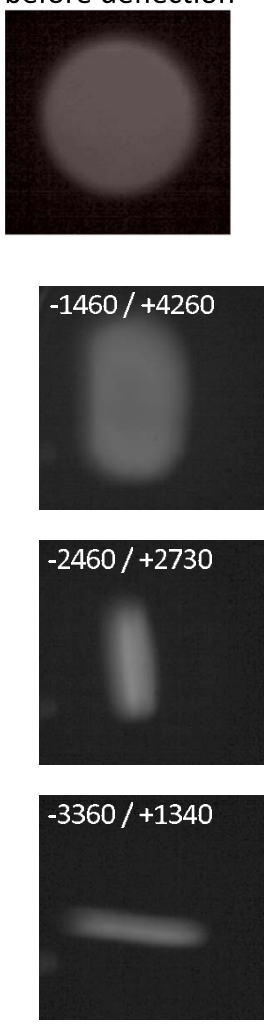
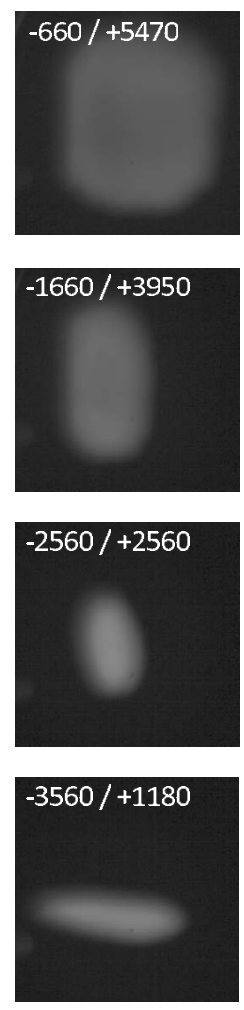
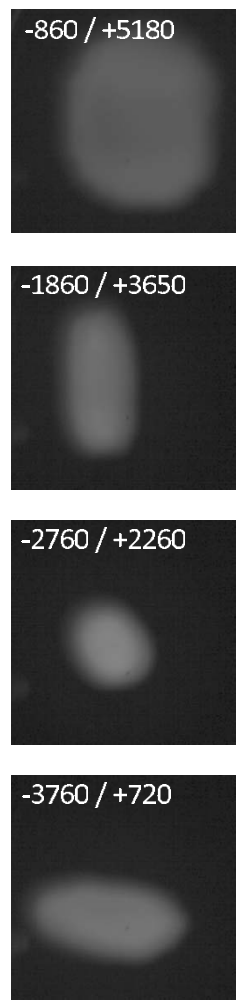
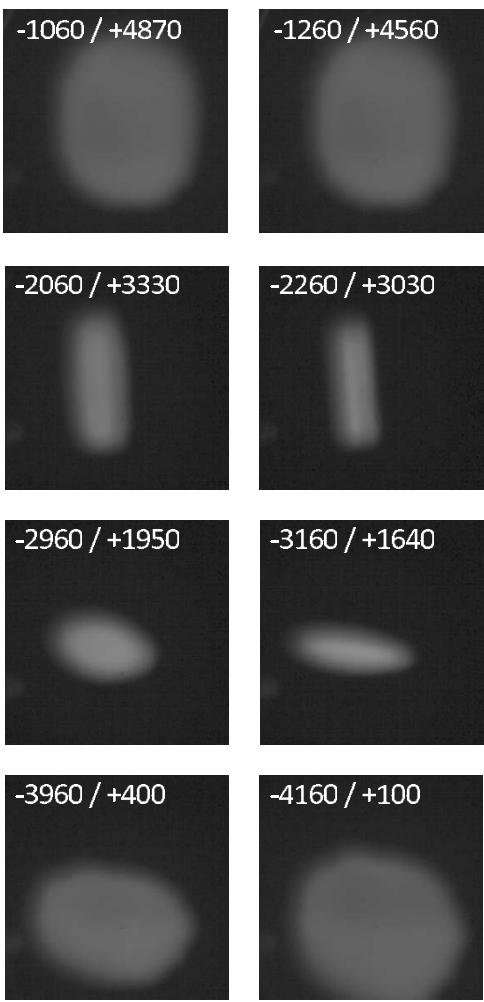
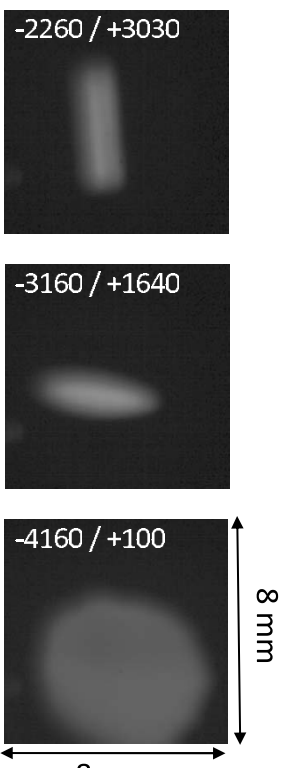

FIG. 6. (Color online) Images of a $10 \mathrm{keV} \mathrm{H}^{-}$beam after deflection in a deflector with differing plate heights. These measurements were taken to demonstrate the ability to manipulate the beam profile using asymmetric voltages. A simple test version of the deflector was used with an inner (outer) plate radius of 145 $\mathrm{mm}(185 \mathrm{~mm})$ and an inner (outer) plate height of $45 \mathrm{~mm}(101 \mathrm{~mm})$. The initial ion beam was sent through a series of $5 \mathrm{~mm}$ apertures before injection into the deflector, producing a collimated beam with a uniformly filled circular profile (upper left corner). The voltages for the outer/inner electrode are given in the insets. The MCP imaging detector was located $\sim 76 \mathrm{~mm}$ behind the deflector exit aperture. Some of the profiles are cropped at the left-hand side since an area close to the edge of the MCP had to be used in order to avoid a burned-out region in the center of the detector.

detector plane varies from circular patterns to horizontally and vertically oblong shapes. For most of the images, the beam profile displays a diagonal distortion. We attribute this effect to the imperfect alignment of the deflector inside the vacuum chamber. The deflector design that was used for the test measurements was optimized for simplicity rather than precision.

The ability to manipulate the beam profile by applying
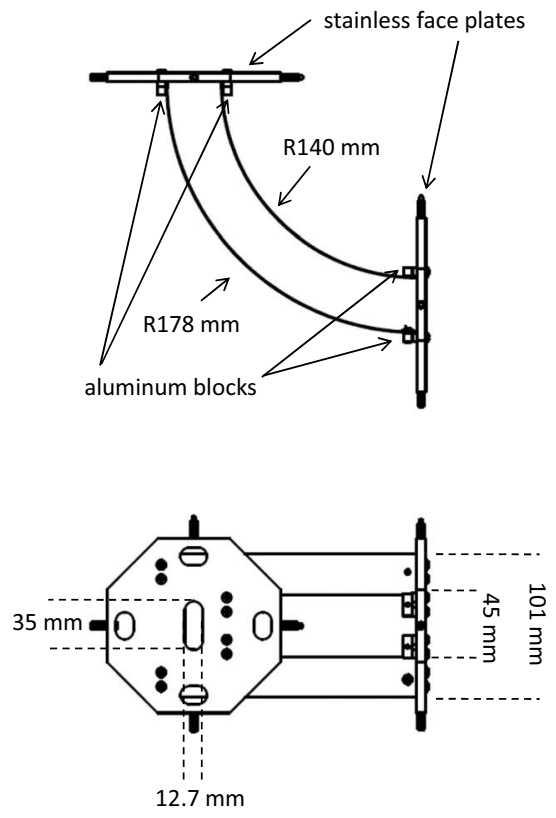

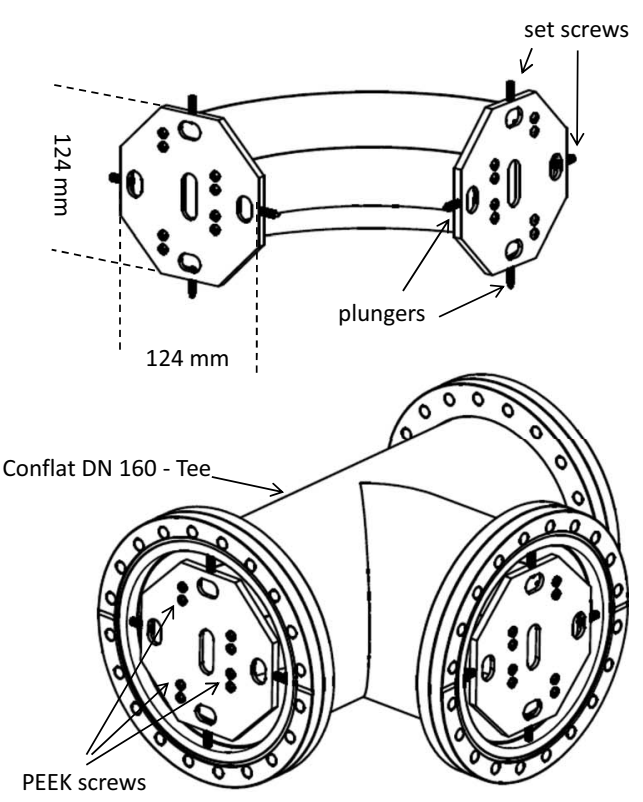

FIG. 7. Mechanical drawings of the cylindrical deflector with differing plate heights. 
asymmetric potentials can be a distinct advantage when working with ion beams that are not necessarily perfectly circular. Test measurements with a discharge ion source at the University of Illinois at Urbana-Champaign have shown that the current density transmitted through circular apertures can be increased by the use of asymmetric voltages. In this case the deflector not only serves to bend the beam at a right angle, it also acts as an ion optical element, optimizing the beam shape.

Following more detailed simulations, the second version of the deflector was built with a revised plate height ratio of $101 \mathrm{~mm} / 45 \mathrm{~mm}$. Figure 7 shows the mechanical drawings of this second version. The electrode plates were manually rolled from flat stainless steel sheet metal. They are held by two stainless face plates with rounded rectangular aperture openings. The mechanical connection between the face plates and the electrodes is provided by small aluminum blocks that are fixed to the face plates with polyether ether ketone (PEEK) screws and ceramic tubes for electrical isolation. The face plates are clamped into the vacuum tube utilizing opposing set screws and nose plungers for ease of adjustment. The electric potentials are fed into the vacuum by safe high voltage (SHV) feedthroughs welded into the vacuum chamber walls and connected to the backsides of an opposing pair of the small aluminum connector blocks. All materials were chosen to be ultrahigh vacuum compatible. Without special precautions the analyzer can be operated with voltages up to $\pm 5500 \mathrm{~V}$ allowing for the deflection of ion beams with energies of $\leq 20 \mathrm{keV}$. Extending this range to higher energies would be possible with simple modifications.

In one implementation we have used a hole in the outer plate to be able to overlap the ion beam with a laser beam. In this configuration it is also possible to switch off the potentials and let the ion beam exit straight through the hole. The beam deflector may thus serve as an ion beam switch and one can easily envision using several consecutive beam deflectors as switches between different beamlines.

\section{CONCLUSION}

A simple electrostatic deflector with focusing properties in two dimensions can be constructed from cylindrical plates with differing plate heights. In combination with an Einzel lens, this deflector allows for the $90^{\circ}$ deflection of a collimated beam with tolerable beam distortions. The use of asymmetric potentials adds flexibility and allows for extensive manipulation of the beam profile. The electric potentials needed for beam deflection are much lower than for a quadrupole deflector at the same beam energy. It is planned to optimize the design in order to be able to work with higher beam energies and to minimize aberrations.

\section{ACKNOWLEDGMENTS}

This work was supported in part by the NSF Division of Chemistry Grant No. CHE-0520660 and Division of Astronomical Sciences Grant Nos. AST-0606960, AST-0807436, and AST-0905832. H.B. was supported in part by the German academic exchange service DAAD. We thank C. C. Havener and $\mathrm{X}$. Urbain for stimulating discussion and $\mathrm{M}$. W. Porambo who helped with some of the SIMION simulations.

${ }^{1}$ J. R. Pierce, Theory and Design of Electron Beams, 2nd ed. (Van Nostrand, New York, 1954).

${ }^{2}$ H. Wollnik, Optics of Charged Particles (Academic, Orlando, 1987).

${ }^{3}$ J. H. Moore, C. C. Davis, and M. A. Coplan, Building Scientific Apparatus, 3rd ed. (Westview, Boulder, 2002).

${ }^{4}$ G. D. Yarnold and H. C. Bolton, J. Sci. Instrum. 26, 38 (1949).

${ }^{5}$ H. Z. Sar-el, Rev. Sci. Instrum. 38, 1210 (1967).

${ }^{6}$ A. L. Hughes and V. Rojanski, Phys. Rev. 34, 284 (1929).

${ }^{7}$ E. M. Purcell, Phys. Rev. 54, 818 (1938).

${ }^{8}$ H. D. Zeman, Rev. Sci. Instrum. 48, 1079 (1977).

${ }^{9}$ M. E. Rudd, in Low Energy Electron Spectrometry, edited by K. D. Sevier (Wiley-Interscience, New York, 1972), Chap. 2.

${ }^{10}$ H. Kreckel, H. Bruhns, and D. W. Savin, U.S. utility patent application serial number 12/708,886.

${ }^{11}$ J. W. Farley, Rev. Sci. Instrum. 56, 1834 (1985).

${ }^{12}$ D. A. Dahl, Int. J. Mass. Spectrom. 200, 3 (2000).

${ }^{13}$ SIMION 3D 8.0, http://www.simion.com (2008).

${ }^{14}$ Here and henceforth, we specify the outer radius of the inner plate and the inner radius of the outer plate since these are relevant for the plate distance and the electrostatic fields.

${ }^{15}$ H. Bruhns, H. Kreckel, K. Miller, M. Lestinky, B. Seredyuk, W. Mitthumsiri, B. L. Schmitt, M. Schnell, X. Urbain, M. L. Rappaport, C. C. Havener, and D. W. Savin, Rev. Sci. Instrum. 81, 013112 (2010). 